\title{
The First Year of Life
}

\section{DISLOCATED AND DISLOCATABLE HIP IN THE NEWBORN}

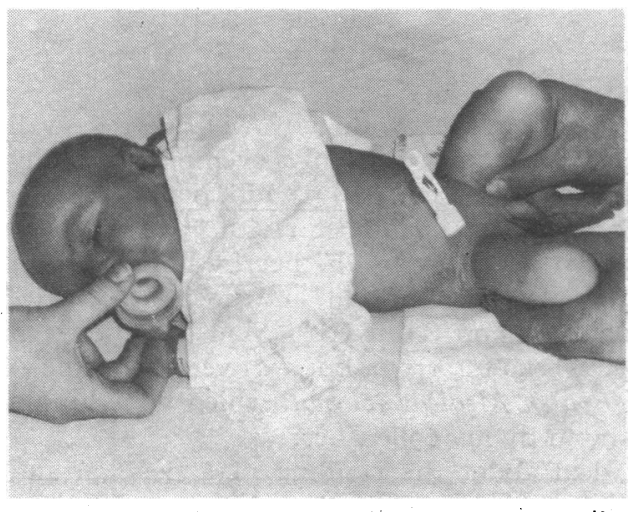

Congenital dislocated hip and dislocatable hip are probably the most important asymptomatic congenital abnormalities to detect, as early treatment is simple and usually effective. In the first two days of life one in 100 infants in Britain has a hip abnormality. Formerly, when they were all left untreated in the newborn, the incidence of established dislocated hip was 1 in 800 children. There may be a family history of the condition; the anomaly is more common in girls and after the breech position in utero. There is a higher incidence in some countries such as Italy and Yugoslavia.

The best time to examine the infant's hips is between the ages of 12 and 36 hours, as the tendency to provoke regurgitation is less by that time. The examiner's hands should be warm and the infant should be placed on her back with the cot clothes spread completely flat. She should be fully relaxed by putting an empty sterile feeding teat in her mouth. The gentle abduction test, followed by Barlow's test, should be carried out in all cases. Unnecessary trauma to the delicate hip joint and its capsule must be avoided.

\section{Gentle abduction test}

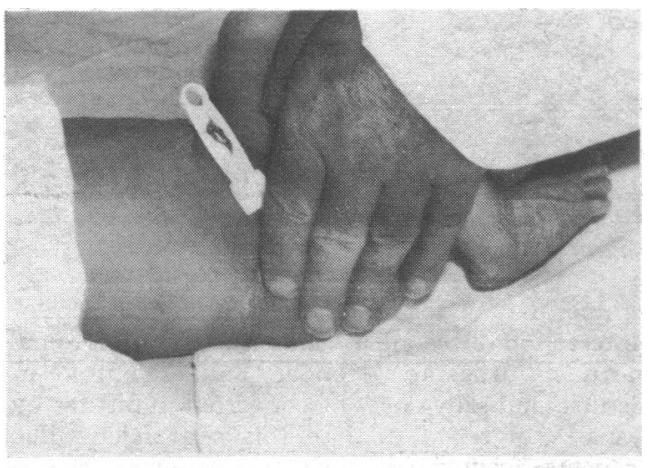

The gentle abduction test will detect hips which are in the dislocated position at rest. Each hip should be examined separately, while the opposite thigh is gently fixed by the examiner's other hand. Both the knee and the hip should be flexed to a right angle and the knee held so that the examiner's thumb is parallel to the medial aspect of the lower thigh, while the middle two fingers lie along the whole length of the lateral aspect of the femur. The tips of the examiner's fingers thus lie over the greater trochanter.

The thigh should be held lightly and neither pushed down towards the cot surface nor pulled up towards the examiner's face. It should then be allowed to abduct very gently and slowly by the weight of the infant's leg until abduction is complete. The thigh should never be forcibly abducted and it is unnecessary to obtain abduction beyond $10^{\circ}$ above the flat. While abducting the thigh, the examiner may feel or see the head of the femur slip, jerk, or jolt forward into the acetabulum. A temporary interruption in the flow of abduction at a point about midway through abduction precedes the sensation of this abnormal movement of the head of the femur.

If the joint capsule is very lax, the reduction jolt may be missed. 


\section{Barlow's test}
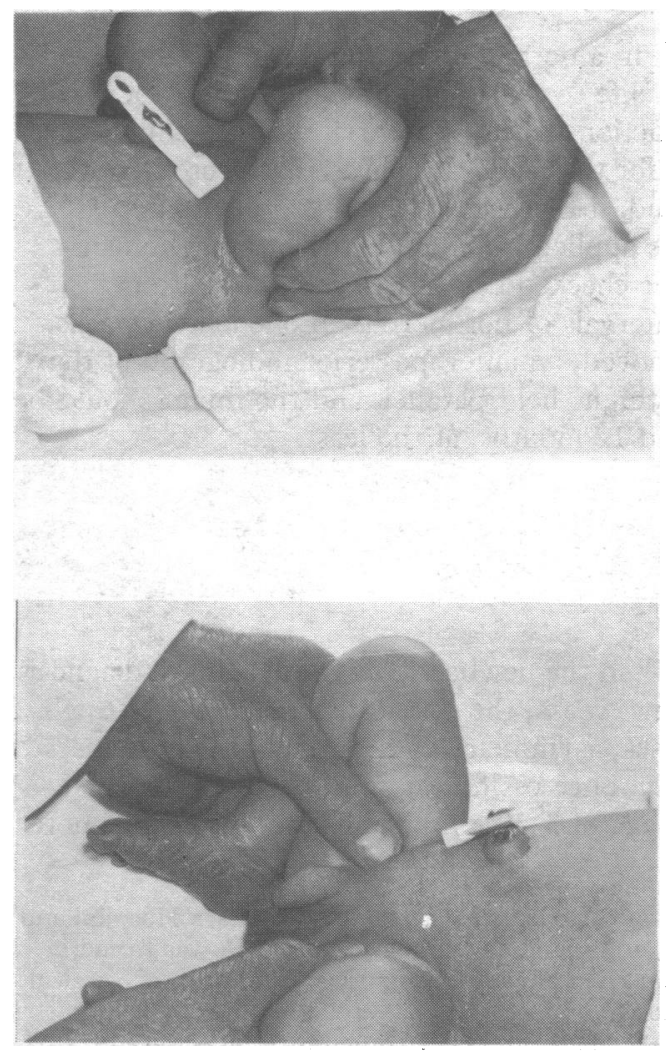

\section{Management}

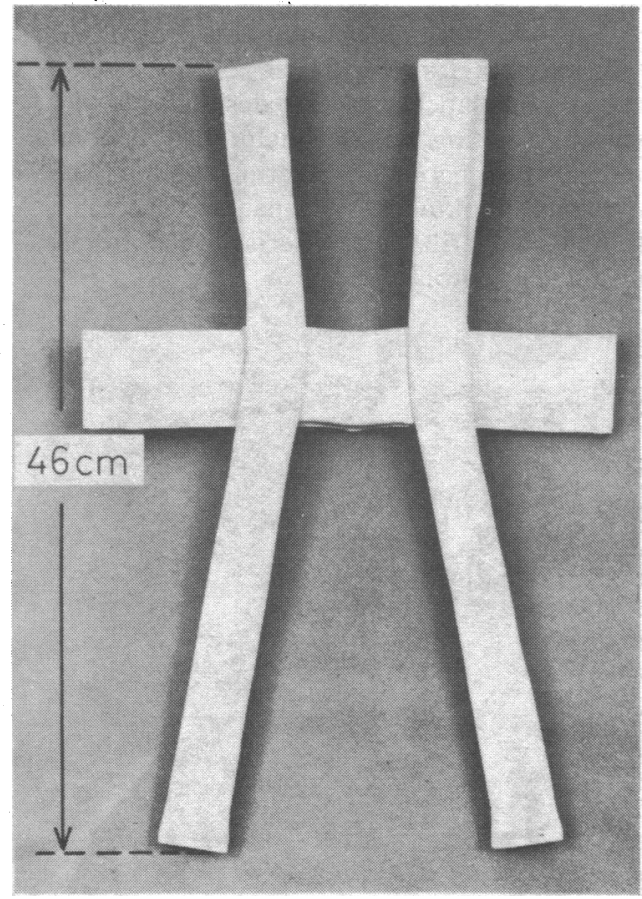

The object of Barlow's test is to identify dislocatable hips in which the head of the femur can be gently jolted posteriorly over the posterior lip of the acetabular labrum to lie temporarily out of the acetabulum and those dislocated hips in which the head of the femur can be jolted forwards to lie temporarily in the acetabulum.

Each hip should again be examined separately while the opposite thigh is gently fixed by the examiner's other hand. The infant's hip should be flexed to a right angle and the knee more acutely flexed. The examiner should place his thumb as high as possible on the medial aspect of the upper femur while the tips of the middle two fingers grasp the greater trochanter laterally.

The thigh is held lightly in a position of only minimal abduction and then an attempt is made to push the femoral head gently posteriorly and slightly superiorly, while at the same time the examiner's hand is internally rotated through not more than $25^{\circ}$. This is followed by reversing the whole movement. No excessive force is used and only a very limited range of movement employed in the test.

The movement of the head of the femur out and in, or in and out, of the acetabulum produces a jolting or jerking movement, which can be seen and felt by the examiner. It cannot be heard by an individual with average hearing. The sensation has been likened to that of a gear lever engaging.

Hips that show excessive movement of the head of the femur within the joint, without being actually dislocatable, are classified as normal.

Ligamentous noise-In at least $10 \%$ of infants the examination described above evokes a noise, click, snap, or grating sensation, but there is no abnormal movement of the femoral head. Such hips should be considered normal and no follow-up is required. Because of past confusion, the term "clicking hip" should be abandoned.
Apart from cases of irreducible hip dislocation, an infant with a dislocated or dislocatable hip should have a splint applied at about the age of 36 hours. Earlier application may be associated with aspiration of mucus or feed, while further delay may cause poorer results. Variants of the von Rosen splint are used: they are made of malleable metal which has been padded and then covered with waterproof material. The splint acts equally on both hips, keeping both thighs flexed and at an optimum degree of abduction. Unless substitution by a larger splint is required, the splint must remain in position continuously for at least two months if the hip is dislocatable, or for at least three months if it is dislocated at rest. Early consultation with an orthopaedic surgeon, who will continue long-term care, is essential.

Before the splint is first applied, the mother must be told exactly what this treatment entails. She should be encouraged to continue breast-feeding, even though this may prove awkward initially. The infant should be placed naked on the splint, which has been fashioned so that the posterior cross bar is grooved to protect the skin over the spine. If the hip is dislocated, the dislocation must first be gently reduced and the thigh held in the abducted position while the splint is carefully applied. Potential pressure points may be protected by inserting pieces of cotton wool or similar material. The mother should be told to replace these when they become wet or soiled without disturbing the splint. The baby's clothes should be put over the splint and not under it. 


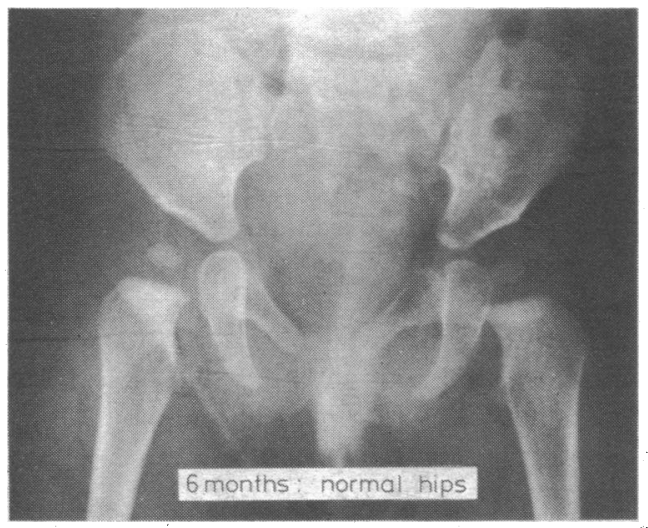

\section{Late diagnosis}

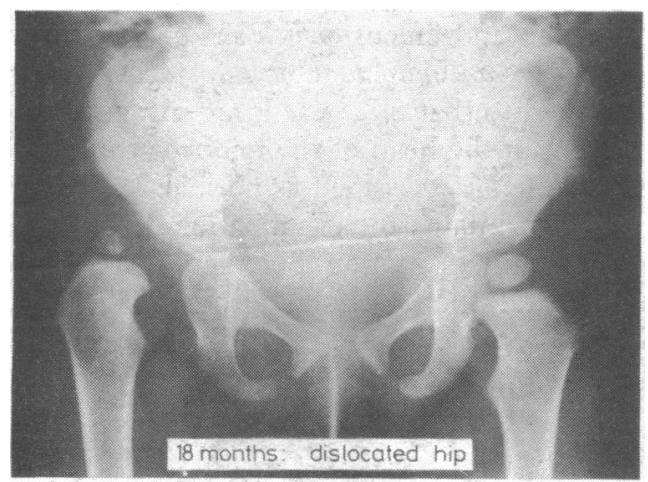

Once the splint is in place, the infant should be washed, weighed, and examined without the splint being removed. The splint will need scrupulous adjustment at each visit to allow for growth and to ensure that the hip is not being overabducted.

The degree of full abduction in a normal infant diminishes slightly but steadily over the early weeks of life so that the splint should give the comfortable degree of full abduction that is appropriate for the child's age. Failure to adjust progressively for this may cause avascular necrosis of the femoral head. On the other hand, if the degree of thigh abduction is inadequate because the splint is applied too loosely, the hip may remain dislocated. The splint should be checked daily for several days after application and thereafter at intervals of not more than one or two weeks.

Once the splint is finally removed, an anteroposterior radiograph of the hips should be taken with the thighs held parallel, and the infant should be followed up at the ages of 6 and 12 months, at the least.

If a dislocated hip is "missed" in the newborn infant, the clinical diagnosis is often difficult until, after some weeks, the classical signs of lack of thigh abduction and, in unilateral cases, asymmetry of the lower buttock creases become apparent. Radiologically, once ossification occurs in the upper femoral epiphysis at about the age of 3-4 months a dislocation can be more clearly shown.

Dr H B Valman, MD, FRCP, is consultant paediatrician, Northwick Park Hospital and Clinical Research Centre, Harrow, and Dr H V L Finlay, FRCP, DCH, was formerly consultant paediatrician, Hillingdon Hospital and Paediatric Cardiothoracic Surgical Unit, Harefield Hospital, Middlesex.

The photographs were taken by Mr E Stride, Department of Medical Photography, Hillingdon Hospital, Middlesex.

\section{Equipment for examining children}
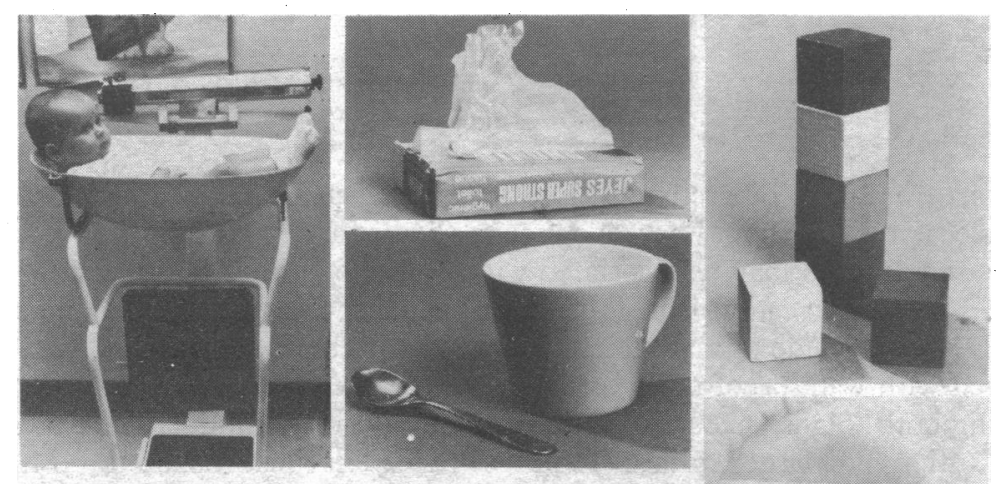

Most of the equipment needed for examining and assessing children is cheap, but its use would greatly improve the quality of care: scales, disposable paper tapemeasure, measuring rod, torch, auriscope, spatula, centile charts, one-inch cubes, Nuffield rattle, cup and spoon, Ladybird first reading books, miniature cups and saucers, small desk and table, pictures on the ceiling, toys (including wooden puzzles, pencils and paper), miniature bell, tissue paper, disposable napkins, urine bags, heel prick equipment including stilet and Steriswab, ophthalmoscope, and cyclopentolate drops.
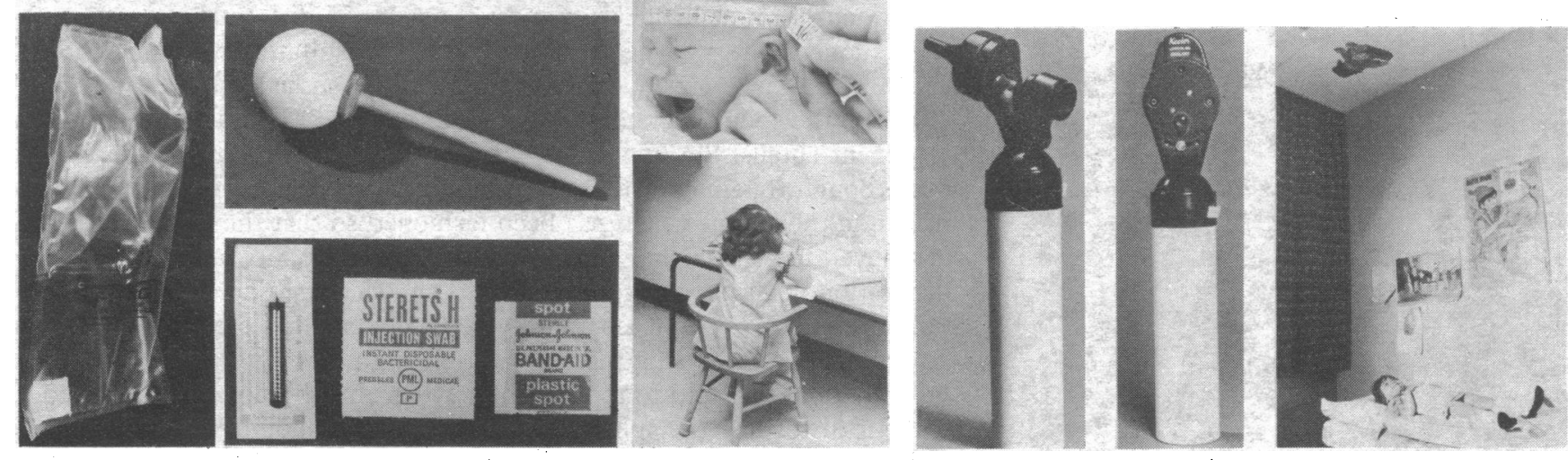